\title{
A System to Depict the Cognitive Process of Trainees Based on Multiple Skill Parameters: System Validation Involving Normal Individuals with an Electrician's
}

\section{License}

Norikatsu Fujita, Hiroshi Takeshita, Sho Aoki, Hirotada Fukae, Kazushige Matsumoto, Tomohiro Murakami and Minoru Hoshino

Ability Development Dept., Polytechnic University of Japan

\begin{abstract}
Instructors of vocational training are increasingly encountering trainees, such as individuals with developmental disorders, who need special accommodations. Flexible teaching of diverse trainees requires the characteristics of trainees to be comprehensively ascertained from an educational perspective. This paper describes a system to depict the cognitive process of trainees based on multiple skill parameters to comprehensively ascertain the characteristics of trainees from an educational perspective. The system to depict the cognitive process while feeding back to the trainee skills is essential for vocational training. Instructors of vocational training will give optimum instruction to each trainees based on the cognitive process generated by the system. From the simulation using the theoretical value and the verification by the field, we clarified that the cognitive process depiction of the trainee can be performed with 32 multi-dimensional skill parameters.
\end{abstract}

Key words: Vocational training, theory of multiple intelligences, action research, M-GTA, developmental disabilities, cognitive information processing model.

\section{Introduction}

In vocational training, trainees in every age group have various vocational and educational backgrounds. Over the past few years, there have been an increasing number of situations where trainees, such as individuals with developmental disorders, need special accommodations. Instructors of vocational training have to teach them in accordance with their abilities.

In general schools for vocational training, a method to learn from causality analysis of successes and failures is used to deal with people with developmental disabilities [1-3]. However, the information obtained from this method is only the causal relationship between factors that affect a

Corresponding authors: Norikatsu Fujita, engineering/ doctoral degree, research field: educational technology. specific situation. Therefore, dealing with various situations encountered in reality is difficult. In other fields, there have been attempts to explore parameters with which to comprehensively ascertain the characteristics of subjects, and there are no parameters available from the viewpoint of education in vocational training [4]. However, parameters with which to comprehensively ascertain developmental disorders are mainly those used for diagnosis [5], and there are no parameters available from the viewpoint of education in vocational training.

Until now, there are training practices that use evaluation scales such as interpersonal skills and social skills [6, 7]. In addition, a system to measure skill gap by measuring physical exercise skill using a haptic device has been reported [8, 9]. However, these efforts target specific skills necessary for vocational training. 
This paper describes a system to depict the cognitive process of trainees based on multiple skill parameters to comprehensively ascertain the characteristics of trainees from an educational perspective. The system to depict the cognitive process while feeding back to the trainees skills is essential for vocational training. Vocational training instructors will give optimum instruction to each trainee based on the cognitive process of the trainee generated by the system.

\section{A Theoretical Model of Teaching Competencies Formation Process of Vocational Training Instructors}

In vocational training, conventional teaching methods do not work, and struggle to respond to trainees who need consideration to cause unexpected reactions and behaviors. From such a problem, the purpose of this study is to realize evidence-based training from the cognitive process of trainees generated by the system. In a previous study, the current authors analyzed the development of teaching competencies in experienced instructors of people with developmental disabilities using a modified grounded theory approach [10].

Fig. 1 shows a theoretical model of teaching competencies formation process of experienced instructors (hereinafter referred to as the theoretical model). This theoretical model was constructed by M-GTA. The M-GTA makes unique modifications to technique, improving GTA [11] for greater practicability, developed by Kinoshita. Unlike GTA, M-GTA does not use technique of slicing data, but uses the concepts of the Analytical Theme and Analytically-Focused Person [12]. M-GTA is an inductive method for creation of a theoretical model, requiring skill and experience in analysis and involving a high degree of difficulty. Dr. Takeshita, one of the authors, is a supervisor of the M-GTA Study Group and has sufficient experience.

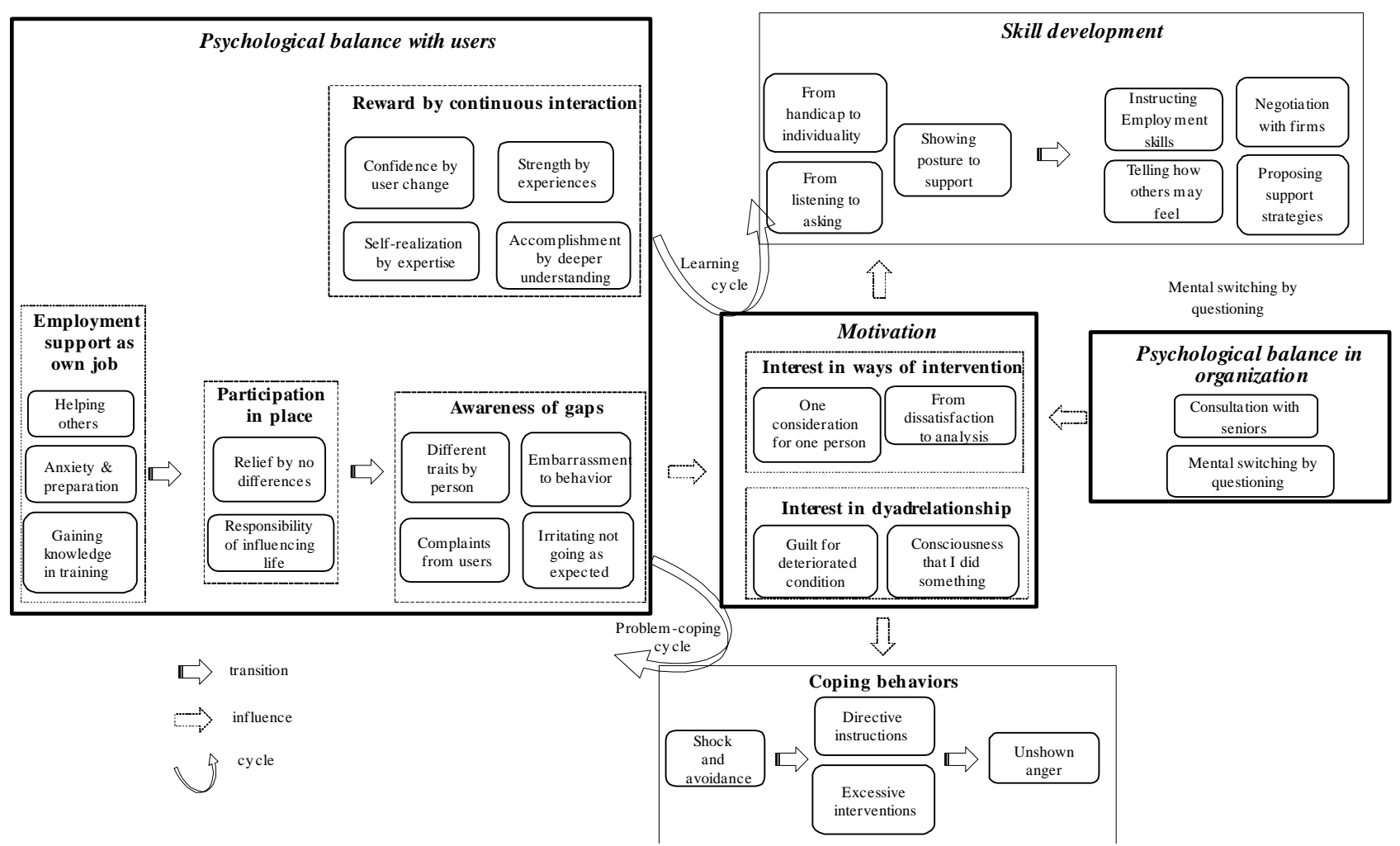

Fig. 1 A theoretical model of teaching competencies formation process of experienced instructors (Details of the concept are shown in Fig. 6.) 
This theoretical model was constructed by semi-structured interview with 15 senior instructors who specialize in teaching people with developmental disabilities. The semi-structured interview was conducted by the interview guide shown in Table 1.

In this theoretical model, Instructors first are interested in investigating the causes of problems dealing with persons with developmental disabilities by recognizing their lack of experience. Then, instructors will "skill formation" against "user" and "organization". The instructors will grow to become an experienced instructor through such "learning cycle". If a problem-coping cycle occurs, instructors become frustrated that trainees fail to meet their expectations and instructors see no way out. The plight of people with developmental disabilities worsens, and instructors who are "Interested in ways of intervention" fall into a Problem-coping cycle as they become "Coping behavior". To escape from such a Problem-coping cycle, it is necessary to make a "Psychological balance in organization" by consultation with seniors and mental switching by questioning. In other words, the theoretical model shows the need to improve the environment of "Psychological balance in organization" and the smooth circulation of "learning cycle".

The basic approach to this study is to adopt an action research methodology involving mutual feedback of "theories" and "practice." This system realizes "skill development" by circulating "learning cycle" in the theoretical model. The multiple skill parameters are a common measure for "skill development". By storing best practices with multiple skill parameters as key, we can construct a training environment based on evidence.

\section{Depiction of the Cognitive Process of Trainees}

In Section 3, we describe depiction of cognitive processes of trainees. The system measures skill factors that cause trainee's unexpected reactions and behaviors from actions selected in a part-time job interview and training at a family restaurant. The trainees will deepen the recognition of his/her skills while receiving feedback on the selected actions. The vocational training instructors decide the overall class guidance and individual guidance from the state of the skill output by the system, and aim to train all participants.

\subsection{Definition of Theoretical Construct}

Depiction of the cognitive process of trainees is constructed by three processes: definition of theoretical constructs, design of questions by stories, and implementation in the system. This section describes definition of the theoretical construct. A theoretical construct abstracts cognitive patterns from human behavior and predicts human behavior.

Fig. 2 shows the theoretical construction based on the cognitive information processing model of Card [13]. The theoretical construct is based on a simple Card cognitive information processing model.

Table 1 Interview guide.

\begin{tabular}{|l|l|}
\hline No & Questions \\
\hline 1 & Please tell me about your current job. (A) responsible work, (b) teaching skills, (c) stakeholders \\
\hline 2 & Please tell me about your experience of employment assistance. (A) yea, (b) content, (c) number of students \\
\hline 3 & Compared to the past, is the concept of trainees dfferent from now? (What triggered the event) \\
\hline 4 & Compared to the past, is the way of teaching trainees different from now? (What triggered the event) \\
\hline 5 & Compared to the past, is the relationship with the trainee different now? \\
\hline 6 & What kind of troubles or problems you have ever had? \\
\hline 7 & What kind of idea did you do? \\
\hline 8 & When do you feel joy, pleasure, rewarding? \\
\hline 9 & In the beginning, what did you most want? \\
\hline 10 & What is the biggest problem at work? \\
\hline
\end{tabular}




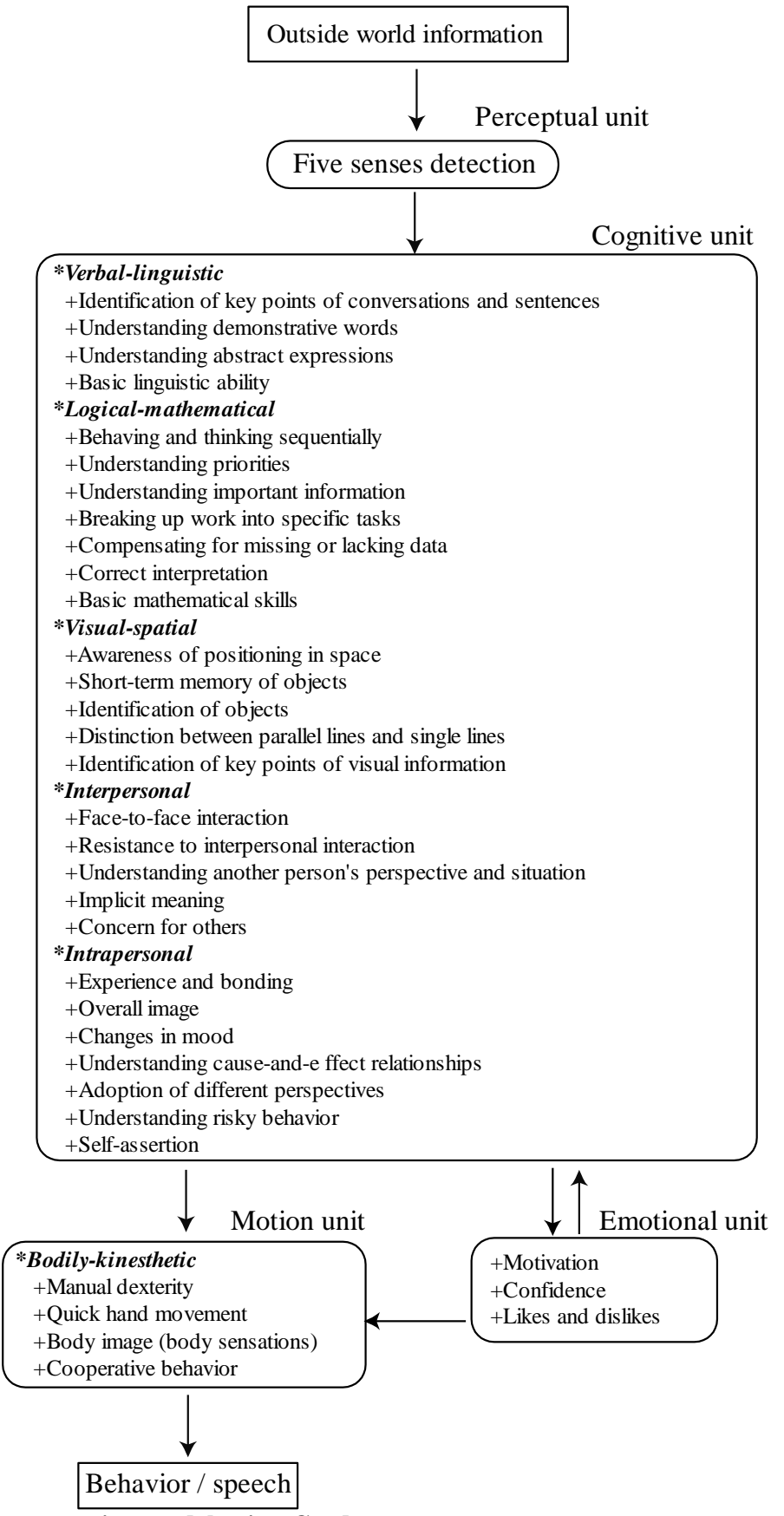

Fig. 2 Cognitive information processing model using Card.

the cognitive information processing model of Card is widely known in the cognitive science field trying to understand the intellectual system and the nature of intelligence from the viewpoint of information processing. Card's cognitive information processing model consists of three systems, "perception system", "cognitive system", and "motor system", and each system includes a memory and a processor. However, since human perception is known to have a deep relationship with psychology, we define a theoretical construct that consists of four systems that add "emotional system". The multiple skill parameters of "cognitive system" and "motor system" are measured by computer. Meanwhile, "perception system" and "emotion system" are measured by instructors by trainee observation. 
The multiple skill parameters are the root of problem behavior in vocational training and are the minimum elements for instructor to develop teaching skills. Many problem behaviors in vocational training are thought to have resulted from a combination of 32 multiple skill parameters.

Extraction of multiple skill parameters is extracted from 192 cases that occurred in the vocational training written in "Support and correspondence guide for trainees who need special consideration" published by Japan Organization for Employment of the Elderly [14]. Dr. Fukae is one of the authors of this book. Multiple skill parameters are extracted based on a theoretical construct by inductive reasoning which considers the cause from the problem behaviors in vocational training. The extracted multiple skill parameters are classified into six categories in the MI theory shown in Table 2. In the MI theory [15], "Naturalistic" "Musical-rhythmic and harmonic" exists in addition to these six categories, but excluded because they are intelligences not related to vocational training. The MI theory advocates that the human frame of mind is 8 intelligences. Educational practices of MI theory are widely practiced in the world [16, 17].

Table 3 shows the details of the multiple skill parameters. The multiple skill parameters consist of 32 in six categories in MI theory. The group index generated by this system represents the category of MI theory. In this way, by classifying the skill factor which is the root of problem behavior by the category of MI theory, we can link with the teaching practice of abundant MI theory. Vocational training instructors can "skill development" with reference to educational practice of MI theory. First of all, the instructor's "skill development" considers teaching methods from the best practice of educational practice of MI theory. Next, consider the more detailed teaching method from the skill situation of the lower multiple skill parameters.

For example, if the group index (5-point evaluation) by Trainee A's MI is below,

Logical-mathematical(4.8)<Visual-Spatial $(4.5)<\mathrm{Bo}$ dily-Kinesthetic(4.0)<Verbal-Linguistic(2.7)

$<$ Interpersonal (2.5) < Intra-personal (2.3)

We refer to best practices of educational practice of MI theory in the order of "Logical-mathematical", "Visual-Spatial", " Bodily-Kinesthetic" which is the good skill of trainee A's. Next, consider the more detailed teaching method from the skill situation of the lower multiple skill parameters. In this way, the system generates a common measure to smoothly circulate the "learning cycle" in the theoretical model. The smooth circulation of the "learning cycle" is also to efficiently train instructors who can give guidance to trainees who need consideration.

\subsection{Design and Implementation of Questions with Story by Cartoon}

Next is design and implementation of questions with story by cartoon. Questionnaires using psychometric measures are commonly used for grasping behavior with psychological phenomena. However, question papers written in language have problems such as dependency on language comprehension degree, difficulty in deeply grasping

Table 2 Multiple skill parameters categories.

\begin{tabular}{|l|l|l|}
\hline Multiple intelligences & Characteristic & Typical example \\
\hline *Verbal-linguistic & Word smart & I like the teaching materials explained in detail. \\
\hline *Logical-mathematical & Number reasoning smart & I like to be reasonably explained. \\
\hline *Visual-spatial & Picture smart & I like to think about while drawing. \\
\hline *Bodily-kinesthetic & Body smart & I like to memorize with the body. \\
\hline *Interpersonal & People smart & I like to learn in groups. \\
\hline *Intrapersonal & Self smart & I like to learn while looking at examples. \\
\hline
\end{tabular}


Table 3 Multiple skill parameters.

\begin{tabular}{|c|c|}
\hline Multiple skill parameters & Typical example \\
\hline $\begin{array}{l}\text { *Verbal-Linguistic } \\
\text { Vl1:Identification of key points of conversations and sentence s } \\
\text { V12:Understanding demonstrative words } \\
\text { Vl3:Understanding abstract expressions } \\
\text { Vl4:Basic linguistic ability }\end{array}$ & $\begin{array}{l}\text { Copying unnecessary information from a blackboard into a notebook. } \\
\text { Only understands words superficially. } \\
\text { Unable to understand sarcasm or indirect references. } \\
\text { Has difficulty reading. }\end{array}$ \\
\hline $\begin{array}{l}\text { *Logical-mathematical } \\
\text { Lm1:Behaving and thinking sequentially } \\
\text { Lm2: Understanding priorities } \\
\text { Lm3: Understanding important information } \\
\text { Lm4: Breaking up work into specific tasks } \\
\text { Lm5:Compensating for missing or lacking data } \\
\text { Lm6: Correct interpretation } \\
\text { Lm7:Basic mathematical skills }\end{array}$ & $\begin{array}{l}\text { Confused by a complicated description of steps involved in wiring. } \\
\text { Unable to understand what to do when instructed to perform several tasks. } \\
\text { Confused when several friends talk at the same time. } \\
\text { Unable to appropriately allocate time when taking a test. } \\
\text { Fails to understand when a conversation is not logical. } \\
\text { Becomes upset after mistaking instruction directed at another } \\
\text { student as intended for oneself. } \\
\text { Has difficulty performing simple calculations. }\end{array}$ \\
\hline $\begin{array}{l}\text { *Visual-spatial } \\
\text { Vs1: Awareness of positioning in space } \\
\text { Vs2: Short-term memory of objects } \\
\text { Vs3: Identification of objects } \\
\text { Vs4: Distinction between parallel lines and single lines } \\
\text { Vs5: Identification of key points of visual information }\end{array}$ & $\begin{array}{l}\text { Collides with objects and desks more often than other students do. } \\
\text { Confused when an instructor returns to a previous slide to explain a point. } \\
\text { Cannot draw a straight line (either by freehand or with drafting equipment). } \\
\text { Cannot clearly draw a double line. } \\
\text { Cannot adeptly hit a ball or shuttlecock with a bat or racket. }\end{array}$ \\
\hline $\begin{array}{l}\text { *Bodily-kinesthetic } \\
\text { Bk1: Manual dexterity } \\
\text { Bk2: Quick hand movement } \\
\text { Bk3: Body image (body sensations) } \\
\text { Bk4: Cooperative behavior }\end{array}$ & $\begin{array}{l}\text { Beautifully written script and notes. } \\
\text { Unable to copy information from the blackboard during class. } \\
\text { Often hits his hand when using a hammer. } \\
\text { Has difficulty choosing clothing appropriate to the season due to hypersensitivity. }\end{array}$ \\
\hline $\begin{array}{l}\text { *Interpersonal } \\
\text { Ie1: Face-to-face interaction } \\
\text { Ie2: Resistance to interpersonal interaction } \\
\text { Ie3: Understanding another person's perspective and situation } \\
\text { Ie4: Tacit understanding } \\
\text { Ie5: Concern for others }\end{array}$ & $\begin{array}{l}\text { Always alone in class. } \\
\text { Agonizes when working in a group or in a lab. } \\
\text { Unable to converse if a friend suddenly strikes up a conversation. } \\
\text { Fails to understand when called on rule violations during a game. } \\
\text { Coolly points out things people may be self -conscious about. }\end{array}$ \\
\hline
\end{tabular}

inner side in linguistic expression, existence of false and socially desirable answers [18]. Therefore, it is difficult to guarantee "reliability" and "validity" in vocational training conducted in various "age", "occupation history", "learning history". Therefore, this system develops a system with a story by cartoon, not questionnaire by language. To set up questions, make the case shown in Table 4 on an episode basis. After that, we implemented questions with a comic story on the system, implemented on the group of subjects to be measured, and repeated individual interviews to ensure practical "reliability" and "validity". This system is not a questionnaire, but a concept model and the case shown in Table 4 is experienced in the context of a part-time job at a family restaurant. By implementing the designed context and questions on the system, forming formative evaluation for the group of subjects to be measured, and repeating individual interviews with the subjects, thereby obtaining "practical" reliability and "validity". The total number of system problems varies depending on the response, and it is small as 24 to 34 subjects. The standard implementation time of the assessment is 20 minutes. The question form is the choice of 5 questions asking actions and the work on the computer. In the question of asking actions by choosing one of the five questions, we always have the option of "I do not know". In addition, although it does not count to the number of questions, as reference information, it is a free description formal, 
and prepares a question "hobby" and "requests from my teacher". Development of the system is made with Articulate Storyline [19] authoring tool for e-learning which is widely prevalent globally.

Fig. 3 shows the number of questions and scores of the system. Previous systems had one question involved with one multiple skill parameter. However, the number of problems was large, the load on subjects was large, and it was also difficult to depict accurate cognitive processes. In the current system, one question involves many multiple skill parameters. That is, by answering one question, many multiple skill parameters are evaluated. Depending on multiple skill parameters, the number of questions involved and the score are different. The skill that is difficult to measure has more questions and the more easily measurable skills reduce the number of questions. Also, there are not only plus but minus points for choice. For example, VII has a problem score of 10 , and a maximum score of 7. The question number of the system and the points of the option are optimized by repeating individual interviews with the measurement subjects. We mentioned earlier that the total number of questions the system displays on trainees is as small as 24 to 34. However, as shown in Fig. 3, the multiple skill parameters are evaluated with the problem of 274 . A major academic achievement of this research is that it showed that the cognitive processes of trainees who need consideration can be depicted with 34 questions (total number of questions 274 in Fig. 3).

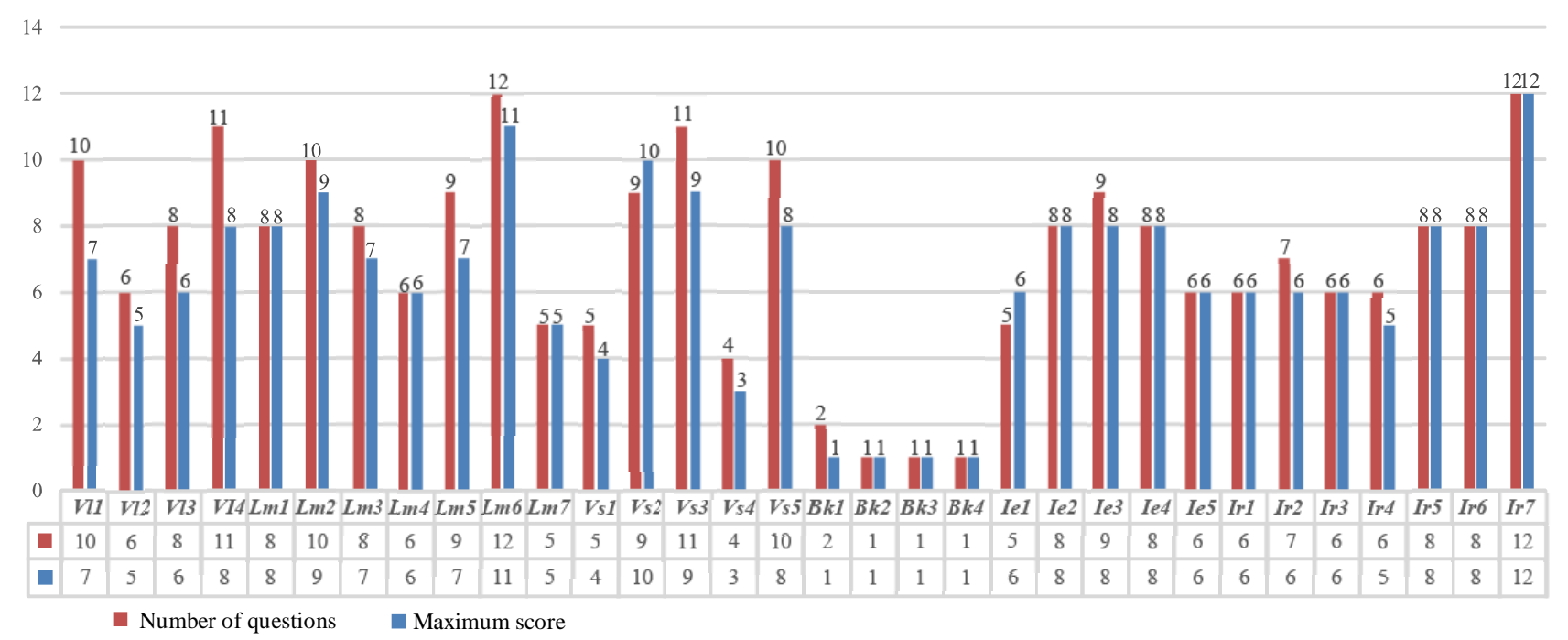

Fig. 3 Number of questions and scores of the system.

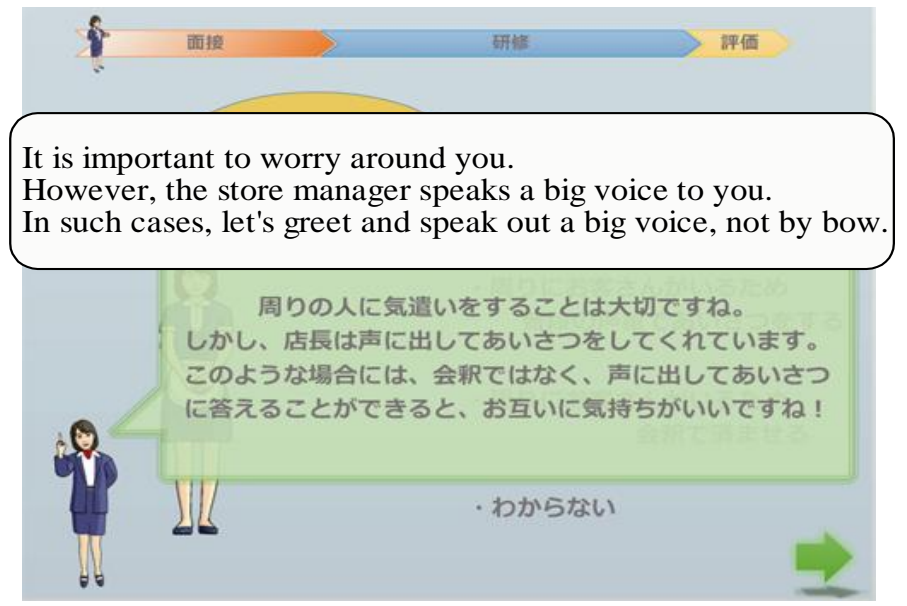

Fig. 4 An example of feedback to the trainees. 
Fig. 4 shows an example of feedback to the trainee. This example is the scene of the first greeting to the manager with part-time job interview. People differ in behavior depending on skill characteristics.

For example, some people greet themselves with a small voice, paying attention to people around outside, others always say with a big voice regardless of the surrounding circumstances. That is, the skill characteristics are related to the actions to be selected. The system depicts the cognitive process of the trainees based on which skills are emphasized. After the trainees respond, the system will feed back to the socially desirable direction that respects the individuality of the trainees. The role of many feedbacks is to inform trainees of the characteristics of their cognitive processes and to realize training in which both trainee and instructor cooperate.

Fig. 5 shows a question example with a branching structure. This is a scene where the store manager instructs to take an order machine. If you can not take the order machine correctly, it will become a scene where customers waiting for accounting will get angry for slowly working clerks. The multiple skill parameter common to these two scenes is "implient meaning" in the conceptual model of Fig. 2. For trainees who do not have the "implient meaning" skills, guidance based on the behavioral principle of sequential proximity method can be considered using language, gesture, model, and body [20]. Until now, the usefulness of overall guidance design that matches

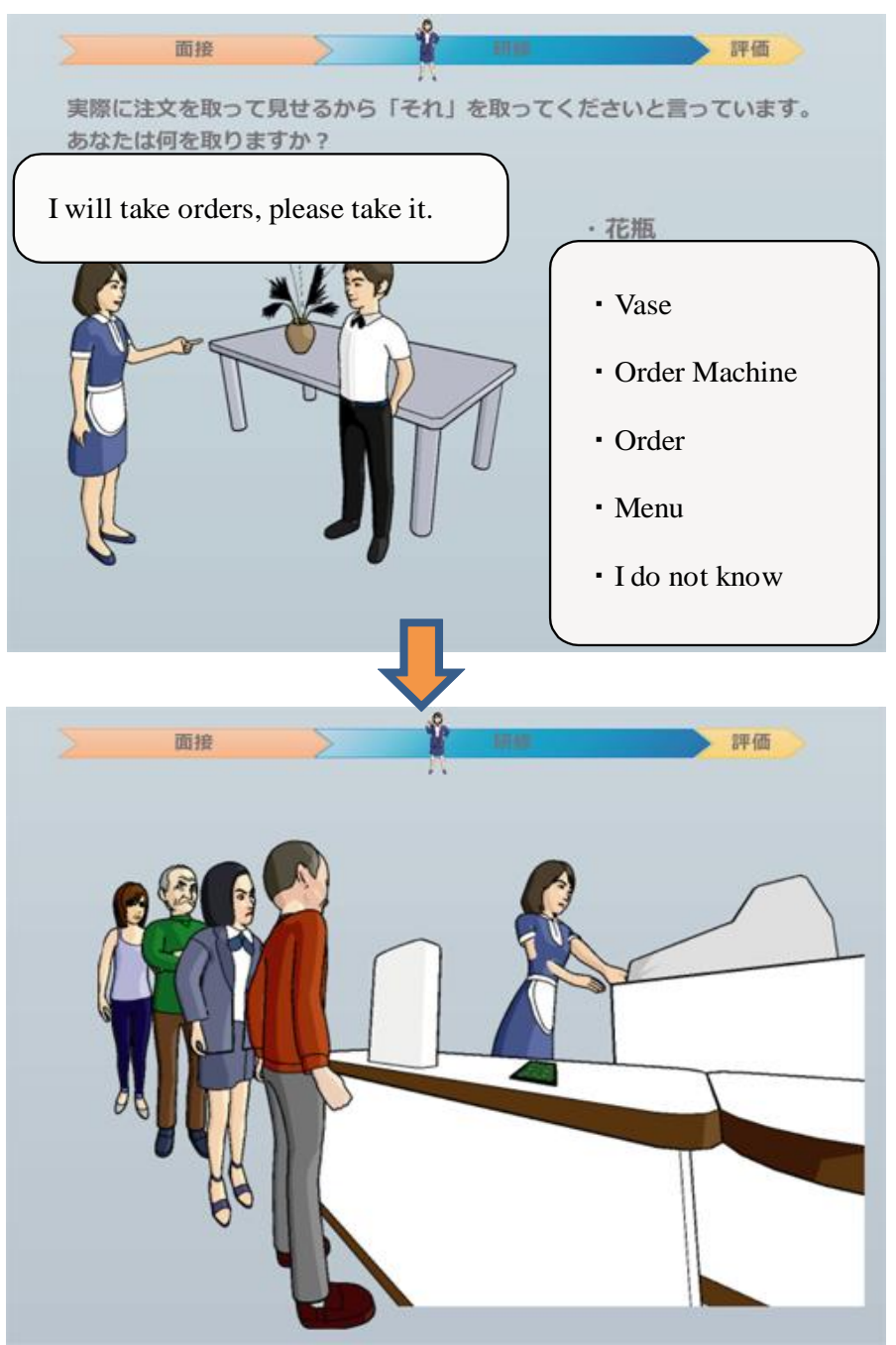

Fig. 5 A question example with a branching structure. 
Table 4 Simulation results of abnormal values using theoretical values.

\begin{tabular}{|c|c|c|c|c|c|c|c|c|c|c|c|c|c|c|c|c|c|c|c|c|c|c|c|c|c|c|c|c|c|c|c|c|}
\hline & Vl1 & VI2 & 73 & $V I 4$ & $\mathrm{Lm}$ & $L_{m 2}$ & 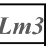 & $\operatorname{Lm} 4$ & $L m 5$ & $\operatorname{Lim} 6$ & $2 m$ & $V s 1$ & $V_{s} 2$ & $V_{s} 3$ & Vs4 & Vs 5 & BkI & $B k 2$ & $B k 3$ & $B k 4$ & Ie1 & Ie2 & Ie3 & Ie 4 & Ie5 & IrI & Ir 2 & Ir 3 & Ir4 & Ir5 & Ir6 & $r 7$ \\
\hline & 5 & 3 & (1) & 5 & 4 & 4 & 4 & 3 & 5 & 5 & 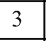 & 4 & 5 & 5 & 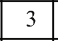 & 5 & \begin{tabular}{|l|l|}
5 \\
\end{tabular} & \begin{tabular}{|l|}
5 \\
\end{tabular} & 5 & 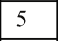 & (2) & 5 & (1) & (1) & 5 & 4 & 4 & 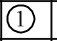 & (2) & (2) & D) & 5 \\
\hline & (2) & (2) & (2) & (2) & (2) & (2) & 3 & 3 & 3 & 3 & & 3 & 4 & 3 & (2) & 5 & 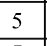 & + & 5 & & 4 & 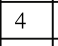 & 3 & 4 & 3 & & (2) & 4 & 3 & 3 & (2) & 4 \\
\hline & 4 & 4 & \begin{tabular}{|l|}
4 \\
\end{tabular} & 4 & 5 & 4 & 5 & 4 & 5 & 4 & & 4 & 4 & 4 & 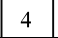 & 5 & (1) & (1) & (1) & (1) & & 4 & 5 & & 4 & & 4 & 4 & & & 4 & 4 \\
\hline
\end{tabular}

Table 5 Traial results.

\begin{tabular}{|l|l|c|c|c|c|c|c|c|c|c|c|c|c|c|c|c|c|c|c|c|c|c|c|c|c|c|c|c|c|c|c|c|}
\hline & $V I 1$ & $V I 2$ & $V l 3$ & $V I 4$ & $L m 1$ & $L m 2$ & $L m 3$ & $L m 4$ & $L m 5$ & $L m 6$ & $L m 7$ & $V s 1$ & $V s 2$ & $V s 3$ & $V s 4$ & $V s 5$ & $B k 1$ & $B k 2$ & $B k 3$ & $B k 4$ & $I e 1$ & $I e 2$ & $I e 3$ & $I e 4$ & $I e 5$ & $I r 1$ & $I r 2$ & $I r 3$ & $I r 4$ & $I r 5$ & $I r 6$ & $I r 7$ \\
\hline $\boldsymbol{D}$ & 4 & 4 & 4 & 4 & 4 & 5 & 3 & 4 & 4 & 3 & 4 & 5 & 3 & 3 & 3 & 4 & 5 & 5 & 5 & 5 & 5 & 5 & 5 & 4 & 4 & 4 & 4 & 4 & 3 & 3 & 3 & 4 \\
\hline $\boldsymbol{E}$ & 4 & 5 & 4 & 4 & 3 & 4 & 3 & 3 & 4 & 4 & 4 & 5 & 3 & 4 & 4 & 4 & 5 & 5 & 5 & 5 & 4 & 3 & 4 & 3 & 4 & 4 & 4 & 4 & 3 & 4 & 3 & 5 \\
\hline $\boldsymbol{F}$ & 4 & 3 & 3 & 4 & 4 & 4 & 3 & 4 & 4 & 3 & 4 & 3 & 4 & 5 & 5 & 5 & 5 & 5 & 5 & 5 & 5 & 4 & 5 & 4 & 4 & 4 & 5 & 4 & 3 & 4 & 4 & 5 \\
\hline $\boldsymbol{G}$ & 4 & 5 & 4 & 4 & 4 & 4 & 5 & 4 & 5 & 5 & 4 & 5 & 4 & 4 & 4 & 5 & 5 & 5 & 5 & 5 & 5 & 3 & 4 & 4 & 4 & 4 & 5 & 5 & 5 & 5 & 4 & 5 \\
\hline $\boldsymbol{H}$ & 4 & 5 & 5 & 4 & 4 & 4 & 4 & 4 & 5 & 4 & 4 & 5 & 3 & 4 & 4 & 5 & 5 & 5 & 5 & 5 & 5 & 5 & 5 & 5 & 4 & 4 & 5 & 4 & 3 & 4 & 4 & 5 \\
\hline $\boldsymbol{I}$ & 4 & 4 & 4 & 4 & 4 & 4 & 5 & 4 & 5 & 4 & 3 & 4 & 5 & 3 & 4 & 4 & 5 & 5 & 5 & 5 & 4 & 3 & 5 & 4 & 4 & 4 & 5 & 4 & 5 & 4 & 4 & 5 \\
\hline $\boldsymbol{J}$ & 4 & 5 & 2 & 4 & 4 & 4 & 5 & 4 & 5 & 4 & 4 & 4 & 5 & 4 & 4 & 4 & 5 & 5 & 5 & 5 & 4 & 3 & 4 & 4 & 5 & 5 & 5 & 4 & 5 & 3 & 4 & 5 \\
\hline $\boldsymbol{K}$ & 3 & 2 & 1 & 5 & 5 & 5 & 2 & 5 & 1 & 3 & 5 & 1 & 3 & 4 & 4 & 2 & 5 & 5 & 5 & 5 & 4 & 4 & 3 & 3 & 4 & 4 & 4 & 2 & 3 & 2 & 3 & 3 \\
\hline $\boldsymbol{L}$ & 5 & 5 & 4 & 4 & 4 & 4 & 4 & 4 & 5 & 5 & 3 & 4 & 4 & 3 & 4 & 4 & 5 & 5 & 5 & 5 & 4 & 4 & 4 & 4 & 4 & 4 & 5 & 4 & 3 & 4 & 5 & 5 \\
\hline $\boldsymbol{M}$ & 5 & 5 & 5 & 4 & 4 & 5 & 5 & 4 & 4 & 4 & 4 & 4 & 5 & 4 & 4 & 4 & 5 & 5 & 5 & 5 & 4 & 4 & 4 & 4 & 4 & 4 & 4 & 4 & 5 & 4 & 4 & 5 \\
\hline
\end{tabular}

\begin{tabular}{|c|c|c|}
\hline No & Concept & Definition (This phenomenon is that ...) \\
\hline & From diss atisfaction to analysis & A staff considers the causes of communication failure, without accusing the user. \\
\hline & Guilt for deteriorated cond ition & A staff feels respon sibility because his/her intervention led user cond ition deterioration. \\
\hline & Anxiety \& preparation & A staff thinks facing with users' disability traits as a part of own job. \\
\hline & Self-realization through expertise & A staff utilizes their experiences on their welfare and business practice through suppo rting users. \\
\hline & Helping others & A staff find s value in supporting other s life. \\
\hline 6 & Shock and avoidance & A staff is shocked by user s background and trying to leave the user to his colleagues. \\
\hline & From hand icap to ind ividuality & A staff accepts the user s disability traits as ind ividuality. \\
\hline 8 & From listening to asking & A staff receives the user $\mathrm{s}$ reaction and asks the cause and background. \\
\hline & Unshown ang er & A staff does not take attitude even if feels ang er in the user s behavior, and diss atisfaction is accumulated \\
\hline 10 & Consciousness that I did something & A staff feels sad by thinking that he/she did something for the user but the user did not respond ed. \\
\hline 11 & Accomplishment by deeper und erstand ing & A staff feels accomplishment by und erstand ing the background of a user s behavior. \\
\hline 12 & Awareness of gaps & A staff no tices the gap between the go als and the reality. \\
\hline 13 & Consultation with seniors & A staff can ask a senior whenever he / she can not und erstand the user s behavior. \\
\hline 14 & Relief by no differences & A staff pleas antly surprises because the users' behaviors do not differ from his / hers. \\
\hline 15 & One consideration for one person & A staff no tices that each user needs one consideration. \\
\hline 16 & Embarrass ment to behavior & A staff is surprised by the sudd en behavior of user and feel uncomfortable. \\
\hline 17 & Irritating not go ing as expected & A staff is irritated and exhausted, as having no sign of improvement of user s cond ition. \\
\hline 18 & Telling how others may feel & A staff tell a user ho w the user $\mathrm{s}$ behavior is seen from the third party. \\
\hline 19 & Mental switching by questioning & By being asked by seniors about the cause of user $\mathrm{s}$ behavior, frustration willswitch to questioning. \\
\hline 20 & Gaining kno wledge in training & A business-experienced staff learns basic kno wledge of suppo rting persons with disabilities in training. \\
\hline 21 & Propo sing suppo rt strategies & A mid-level staff creates and propo ses suppo rt plans each other in various viewpoints. \\
\hline 22 & Confidence by user change & A staff gains self-confidence by the prog ress of users. \\
\hline 23 & Respon sibility of influencing life & A staff feels respon sible because his / her intervention influences the employment (life) of users. \\
\hline 24 & Sho wing posture to suppo rt & A staff lets the user disclose though ts and circumstances by sho wing posture to suppo rt. \\
\hline 25 & Instructing Employ ment skills & A business-experienced staff trains necess ary skills for employment to users. \\
\hline 26 & Streng th by experiences & By having experienced, a staff will not be upset by the disastrous background of ind ividual users. \\
\hline 27 & Different traits by person & Since the situation of users is different for each person, basic kno wledge is not applicable. \\
\hline 28 & Negotiation with firms & A staff can nego tiate with the company about employment of users. \\
\hline 29 & Directive instructions & A staff instructs and requests at the ordinary workplace level to a user. \\
\hline
\end{tabular}

Fig. 6 Details of the concept.

the skill characteristics of individual classes has been confirmed in practical cases of active learning [21] using Just-In-Time Teaching [22]. In this way, this system is useful for both "overall guidance of class" and "individual guidance".

\section{Evaluation}

As system evaluation, from the simulation using the theoretical value and the verification by the field, confirm depiction of the cognitive process of the 
trainee through 32 multi-dimensional skill parameters. This study was approved by the Ethical Committee of the Polytechnic University of Japan (No.607).

Table 4 shows simulation results of abnormal values using theoretical values. The system outputs the evaluation value in five stages. For abnormal value detection, output with evaluation value of 2 or less. The theoretical value $\mathrm{A}$ is a simulation of a trainee who detects an abnormality in a specific skill such as tacit consent. The theoretical value B is a simulation of a trainee who detects an abnormality in the language category and also has another category. The theoretical value $\mathrm{C}$ is a simulation of a trainee who detects abnormality by skill of physical movement category and other category has high skill. From these simulation results, the system is able to output outliers with categories and skills.

Table 5 shows the assessment results of 10 trainees. Subjects are the top trainees in the class who acquired the electrician's license. It is understood that the skill level related to qualification acquisition is uniformly high and there are variations in skills. However, Trainee $\mathrm{H}$ has many items with low skill level. Trainee $\mathrm{H}$ has a low skill level overall. In a later interview, he said that he had worked in a hurry without time. In this system, we emphasized familiarity by embedding context, but it is not perfect.

From the simulation using the theoretical values and the verification by the field, we confirmed that the cognitive process of the trainees can be depicted by the multiple skill parameters defined in this paper.

\section{Conclusion}

In this paper, we described a cognitive process depiction system of trainees based on multiple skill parameters which comprehensively grasp the skills necessary for vocational training from the viewpoint of education. From the simulation using the theoretical values and the verification by the field as described above, we confirmed that the cognitive process of the trainees can be depicted by the multiple skill parameters defined in this paper.

The originality of this work has been to comprehensively ascertain developmental disorders from an educational perspective. This originality is achieved through the followings:

(1) We defined a theoretical construct based on a simple Card cognitive information processing model, which the instructor can easily understand;

(2) We extracted and weighted the multiple skill parameters from the theoretical construct;

(3) We classified the multiple skill parameters in the intellectual category of MI theory from the viewpoint of education in vocational training.

Future tasks are verification for people with developmental disabilities, database construction of educational practice of MI theory, and guidance strategies according to the situation of skill of lower multiple skill parameters.

\section{Acknowledgment}

This work was supported by JSPS KAKENHI Grant Number JP26350303.

\section{References}

[1] Japan Organization for Employment of the Elderly, Persons with Disabilities and Job Seekers, 2010, Research on Promoting Employment of People with Developmental Disabilities Using Technology and Support for Continuing Work. Research Report No.149, Japanese.

[2] Japan Organization for Employment of the Elderly, Persons with Disabilities and Job Seekers, 2007, Effective Vocational Training Examples for People with Developmental Disabilities. Research Report No.119, Japanese.

[3] Japan Organization for Employment of the Elderly, Persons with Disabilities and Job Seekers, 2008, Vocational Training Handbook of People with Developmental Disabilities. Japanese.

[4] Alfred, B., and Jacqueline, C. 1987. "Characterizing Criminal Careers.” Science 237: 985-91.

[5] Kaufman, A. S. 2010. "Lichtenberger, Elizabeth (2006)." In Assessing Adolescent and Adult Intelligence (3rd ed.), Hoboken (NJ): Wiley.

[6] Japan Organization for Employment of the Elderly, Persons with Disabilities and Job Seekers, 2014, Research on Characterization of Communication Skills of 
People with Developmental Disabilities. Research Report No.119, Japanese.

[7] Japan Organization for Employment of the Elderly, Persons with Disabilities and Job Seekers, 2014, Research on Human Resource Development Program Using SST. Research Report No.124, Japanese.

[8] Fujita, N., Hayashi, T., and Yamasaki, T. 2007. "Torch Operation Learning System Based on Welding Skill Parameters." The IEICE Transactions on Information and Systems (Japanese edition) 9: 2522-9.

[9] Aswathi, P., Amritha, N., and Namitha, K. 2016. "Bridging the Skill Gap Using Vocational Training Simulators: Validating Skill Assessment Metrics." In IEEE Conference Proceedings, No.ICACCI, 645-51.

[10] Takeshita, H., and Fujita, N. 2016. "Psychological Intervention in an Employment Transition Support Center: Support Staff Psychological Balance." Poster session presented at the 22nd World Congress of Social Psychiatry-WASP2016, New Delhi.

[11] Glaser, B., and Strauss, A. L. 1967. The Discovery of Grounded Theory. Chicago (IL): Aldine.

[12] Nishigaki, K., 2016. "Changes in Mothers' Psychosocial Perceptions of Technologydependent Children and Adolescents at Home in Japan: Acknowledgement of Children's Autonomy." Asian Nursing Research 10 (2): 100-5.

[13] Card, S. K., and Moran, T. P. 1983. The Psychology of Human-Computer Interaction. Lawrence Erlbaum Associates.

[14] Japan Organization for Employment of the Elderly,
Persons with Disabilities and Job Seekers. 2015. Support/ Correspondence Guide for Students Who Need Special Consideration in the Progress of Training/Learning (practical edition). Japanese.

[15] Gardner, H. 1993. Multiple Intelligences: The Theory in Practice. Basic Books: Reprint Edition.

[16] Gardner, H. 1991. "Assessment in Context: The Alternative to Standardized Testing." In Changing Assrssment: Alternative Views of Aptitude, Achievement, and Instruction, edited by Gifford, B. R., and Connor, M. C. O. Boston: Kluwer.

[17] Gardner, H., Krechevsky, M., Sternberg, R. J., and Okagaki, L. 1994. Intelligence in Context: Enhancing Students' Practical Intelligence for School, edited by K. McGilly, Cambrige: Bradford Books/MIT Press.

[18] Yokouchi, H. 2007. "Basic Understanding of Psychometric Scale.” J. Jpn. So.c Intensive Carw Med. 14: 555-60.

[19] Articlute Storyline. http://www.articulate.com.

[20] Dennis, H., and Reid, C. W. G. 2005. "Preference-Based Teaching: Helping People with Developmental Disabilities Enjoy Learning without Problem Behavior." Habilitative Management Consultants, Inc.

[21] Novak, G. M., and Patterson, E. T. 1999. Just-in-Time Teaching: Blending Active Learning with Web Technology. Addison-Wesley.

[22] Deslauriers, L., Schelew, E., and Wieman, C. 2011. "Improved Learning in a Large-Enrollment Physics Class." Science 332: 862-64. 